\title{
Online Support for Shop-Floor Operators Using Body Movements Tracking
}

\author{
Julien Provost* Amir Hossein Ebrahimi* Knut Åkesson* \\ * Signals and Systems, Chalmers University of Technology, Sweden \\ (e-mail: \{provost;amir.ebrahimi;knut\}@chalmers.se).
}

\begin{abstract}
:
This paper presents results of experimentations on the integration of human operators and automation in the context of assembly systems. Several experiments have been conducted to evaluate the use of augmented reality applications in order to provide dynamic work instructions and online feedback information to a shop-floor operator. Based on these results, an experimental platform has been developed so that the shop-floor operator and the automated system behave as a closed-loop system.
\end{abstract}

Keywords: Augmented reality, Work instructions, Closed-loop system, Body tracking

\section{INTRODUCTION}

In the context of assembly systems - where many operations are done by human operators - important issues are system flexibility, human utilization, and perceived quality of working life. The solution presented in this paper is a way to support the human operator, in order to be more flexible but also to work in a way that is better for the human body. Indeed, high flexibility and high utilization are mandatory to meet the increasing demands on mass customization and reduced time-to-market. Meanwhile, sustainable human resource policies aim at improving employment and quality of working life for human operators. In practice, these issues are not independent; humans are intrinsically more flexible than machines while automation permits to increase safety, efficiency and accuracy. However, the integration of human operators in automated systems remains an issue.

A better integration of human operators in automated systems can be achieved by two means:

- A better instructions' and information's support for the human operator

- A better observation of the human operator's actions

First, even though studies have shown that dynamic instructions are superior to static instructions (Höffler and Leutner [2007]), in industry many instructions are still paper-based (Greenough and Tjahjono [2007]).

Secondly, while an automated system is composed of many sensors that can be scanned with a very short cycle time (down to few milliseconds); the current status of operations performed by human operators may only be updated with a longer cycle time (up to several minutes). These are the sources of outdated information and long communication delays.

Recent studies on augmented reality have investigated different solutions to assist a human operator in the contexts of assembly (Vignais et al. [2012], Iliano et al.
[2012]) and maintenance (Henderson and Feiner [2011], De Crescenzio et al. [2011]). In these studies, the observation of the operator's actions permits either to make sure the operator is working in an ergonomic way or to verify the work-flow of the actions performed by the operator.

The work presented in this paper focuses on the manual assembly context. The solution that is presented permits to provide dynamic instructions and feedback information to the shop-floor operator based on the observation of the shop-floor operator's actions.

In order to investigate these issues, a lab-platform has been developed and different set-up have been evaluated. These evaluations have permitted to select a set-up that allows a better integration of a shop-floor operator with the automated system. As a result, the shop-floor operator and the automated system behave as a closed-loop system. By analogy to fully automated systems, the work instructions displayed to the shop-floor operator can be considered as her/his inputs while the actions that can be observed through a tracking system can be considered as her/his outputs.

The investigations presented in this paper focus on the evaluation of camera-based tracking solutions. The use of both $2 \mathrm{D}$ and $3 \mathrm{D}$ cameras is investigated. The tracking system should be able to detect the operator hands' movements in real-time. Then, thanks to this real-time detection, real-time work instructions can be displayed to the human operator. This permits both to reduce the amount of information that is displayed at a time, and to increase the relevance of this information. This could also permit to provide immediate feedback about the success or failure of the current operation.

The next section presents how an enhanced observation of the operator's movements permits to achieve a better integration of human operators in automated systems. Section 3 presents the comparison of two experimental setups. Section 4 presents the results of a experiment on the relevance of the work instructions. Then, section 5 details 
the application of these results to the selected platform. Finally, section 6 gives an overview of current practices and provides recommendations regarding ethical aspects and user-acceptance of human-body tracking systems.

\section{LEVELS OF OBSERVATION OF SHOP-FLOOR OPERATOR'S ACTIONS}

The interaction between human and automation often refers to the definition of the Levels of Automation (Parasuraman et al. [2000]). This section focuses on the automation of information acquisition applied to the sensing of shop-floor operators' actions.

Indeed, the range of human actions that can be automatically observed is highly dependent on the technology which is used to capture and observe the behavior of a (semi-automated) system. As summarized in the figure 1, the more a system is observable, the more data can be exchanged with the automation system and the shorter will be the average cycle time between two observations.

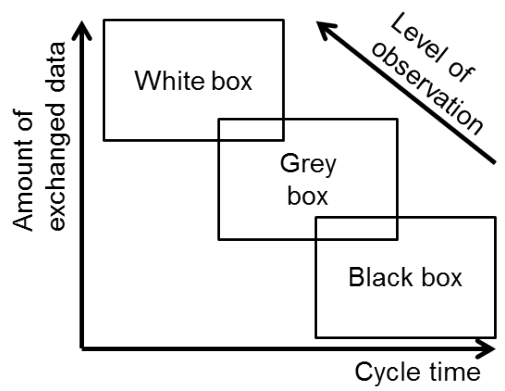

Fig. 1. Levels of observation

As illustrated in the figure 1, three levels of observation can be defined: black-box, gray-box and white-box observation. The following paragraphs illustrate the main difference between these levels through the example of a manual assembly station.

At the lowest level of observation (black-box), the behavior of the station can only be observed, and as a consequence measured, through its inputs and outputs. At this level, nothing of what is happening in the manual assembly station can be observed. Examples of measurements that can be performed on such a station are:

- Throughput: measured at the output of the station or at the input of the next station

- Quality ratio: measured later, once the product has been inspected

Moreover, in the case of a black-box observation, these measurements are not available in real-time (e.g. the inspection of a product can be done several stations later). As a consequence, the whole system behaves with a huge inertia, and is poorly reactive. The relevance of the feedback information provided to the shop-floor operator can also be questioned since this information may be outdated.

At the intermediate level of observation (gray-box), few actions that are executed in the manual assembly station can be observed. For example, this level of observation can be reached by the implementation of push buttons, barcode readers, RFID tag readers, ... so that the shop-floor operator can intentionally interact with the automation system. The integration of the shop-floor operator in the automation system is improved and dynamic instructions can be considered (e.g. step-by-step instructions... ). However, the interactions between the automation system and the shop-floor operator (e.g. scanning a bar-code) is time consuming and corresponds to non value-adding actions.

At the highest level of integration (white-box), the assembly station is "fully observable" by the automation system. The shop-floor operator is immersed in the automation system, her/his position can be detected to avoid collision with moving robots (Lenz et al. [2012]), to assess working ergonomics (Vignais et al. [2012]) or to analyze the workflow of executed assembly operations (Damen et al. [2012]). Regarding the work-flow analysis of the assembly operations, the hands of the operator can be tracked to detect what (s)he is currently doing (picking a part from a box, using a screwdriver...). Thus, instructions and relevant information can be displayed in real-time. At this level, the measurements of the operator performance are more detailed and the feedback information can be provided in real-time (e.g. "You have forgotten to pick a part from box XX", "You are working too fast - next buffer is full" ...).

\section{TRACKING SHOP-FLOOR OPERATOR'S ACTIONS: COMPARISON OF TWO SET-UPS}

This section presents two experimental set-ups that permit to reach the highest level of integration. In the remainder of this paper, the term "fully observable" refers to full observation of the interaction of the shop-floor operator's hands with predefined $3 \mathrm{D}$ areas.

Recent technological advances have permitted the development of wireless sensors and miniature cameras which can be worn on the body (e.g. Stricker and Bleser [2012]). However, in order to increase end-users' acceptance, this paper only considers solutions without on-body sensors.

\subsection{Evaluation of two set-ups}

The tracking system should satisfy the following requirements:

- Automatic detection and tracking of the shop-floor operator's hands;

- No marker or active sensor should be put on the shopfloor operator's body;

- No active sensor should be put on the boxes;

- Processing should be done in real-time;

These requirements could also be applied in the context of human-robot collaboration (Lenz et al. [2012], Tan and Arai [2011]).

The first requirement permits to focus on the hand interaction and to abstract other data. As a comparison, the solution proposed in Lenz et al. [2012] focuses on the safety aspect of human-robot collaborations where the whole operator's body should be detected but not necessary identified. The second and third requirements are necessary to guarantee a flexible system and natural interaction. As a comparison, the solution proposed in 
Iliano et al. [2012] - which requires infrared sensors to be installed on each box - is too complex to update and maintain when the assembly station is modified. The last requirement is mandatory to provide online instructions to the shop-floor operator and up-to-date information.

The next sections present two experiments that have been conducted to evaluate the usability of both $2 \mathrm{D}$ and $3 \mathrm{D}$ cameras to capture the operator's hands movements. Both experiments were conducted on a manual assembly and do not use any on-body marker nor active sensor.

\subsection{First set-up: five static cameras and one head-mounted camera}

In order to evaluate the potential amount of data that can be extracted from static and head-mounted camera, a first experimental platform has been developed. This platform is composed of:

- five static $2 \mathrm{D}$ cameras;

- one head-mounted 2D camera.

The figure 2 illustrates the positioning of these cameras. Three static cameras are placed on top of the assembly station (cameras 1, 2 and 3); two static cameras are also placed two meters away on each side of the assembly station (cameras 4 and 5). The last camera (6) is headmounted; its orientation is set up to correspond to the natural field of view of the shop-floor operator.

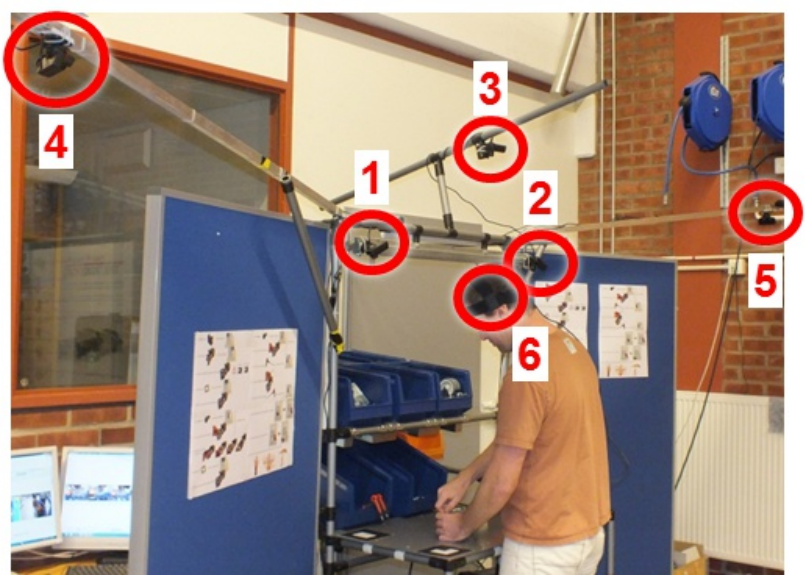

Fig. 2. Illustration of the set-up with 6 cameras

This experiment was made using PlayStation Eye cameras $^{1}$. These cameras were chosen because of their low distortion and decent field-of-view ${ }^{2}$. The figure 3 is a screen-shot of the video captured with this set-up.

First, the video shows that the observation of the scene using only one head-mounted camera is too limited to be able to use only this camera. For instance, on the screen-shot the operator is assembling and looking at the product on the table but her/his hands do not appear on the camera; thus, hands movements cannot be detected. Furthermore,

\footnotetext{
1 http://us.playstation.com/ps3/accessories/playstation-eyecamera-ps3.html. PlayStation Eye specifications. Last visited: 2013-02-27

2 The diagonal field-of-view (FoV) of these cameras is equal to 75 degrees, thus according to the camera resolution the horizontal FoV is equal to 63 degrees and the vertical FoV is equal to 49 degrees
}

the direction of a head-mounted camera is defined by the direction of the user's head. As a consequence, a limited field-of-view combined with a limited neck bending - according to ergonomics recommendations, the range of a human neck bending should be between 0 and 20 degrees (between 0 and 10 is even better) McAtamney and Corlett [1993] - would definitively not be sufficient to be able to capture each and every hand movement of the shop-floor operator.

Secondly, regarding the five static 2D cameras, the use of the three cameras placed on top of the station permits to have a good observation of the operator's hand movements. It should be reminded that at least two $2 \mathrm{D}$ cameras are needed to be able to calculate the position of the hands in the 3D space. However, the lack of out-of-thebox and real-time solutions for the integration of $2 \mathrm{D}$ video for marker-less hand tracking would require an important investment to obtain a fully operational platform. For further details about the evaluation of hand tracking in an industrial context using multiple $2 \mathrm{D}$ videos the reader is referred to Yun et al. [2013].

\subsection{Second set-up: one static 3D camera}

Compared to 2D video tracking, the commercial and opensource offer for body tracking using $3 \mathrm{D}$ video is much more important. Indeed, the video-game industry has impulsed an important dynamic in the development of virtual reality and augmented reality applications. For those reasons, a second experimental platform has been developed in order to evaluate:

- the usability of commercial 3D camera to track the operator hand's movements in an industrial context;

- the usability of video game interface to display work instructions and feedback information.

The first point is particularly important in this evaluation. Indeed, contrary to virtual reality applications - where the cameras can be placed anywhere in the 3D space and the scene is free of obstacle between the cameras and the user - in the case of this platform, the camera should be integrated within a real assembly station and the shopfloor operator interacts with real objects.

This experiment has been conducted on the same assembly station as presented before. For this experiment, the scene is captured using one $3 \mathrm{D}$ camera (both Microsoft Kinect $^{3}$ and Asus Xtion ${ }^{4}$ depth cameras were tested). As depicted in the figure 4 , the $3 \mathrm{D}$ camera is positioned two meters away on one side of the assembly station. The position and orientation of the camera are set up so that the whole body of the shop-floor operator can be detected. The work instructions and a virtual representation of the assembly station are displayed on a screen using the Unity $3 \mathrm{D}$ game development software ${ }^{5}$. For more mobility, this user interface will be replaced by a head-mounted display in a future version.

\footnotetext{
3 http://www.microsoft.com/en-us/kinectforwindows. Microsoft Kinect specifications. Last visited: 2013-02-27

4 http://www.asus.com/Multimedia/Xtion_PRO_LIVE. Asus Xtion specifications. Last visited: 2013-02-27

5 http://unity3d.com. Unity3D video-game software. Last visited: 2013-02-27
} 


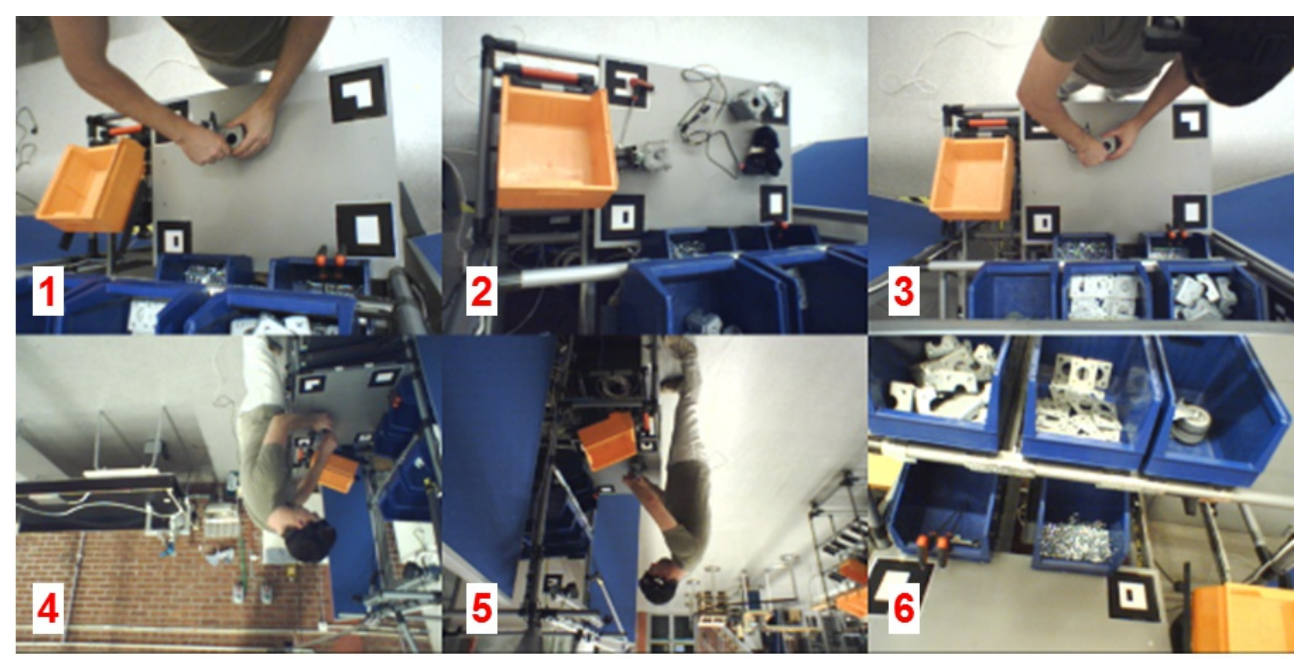

Fig. 3. Screen-shot of the video obtained with 6 cameras

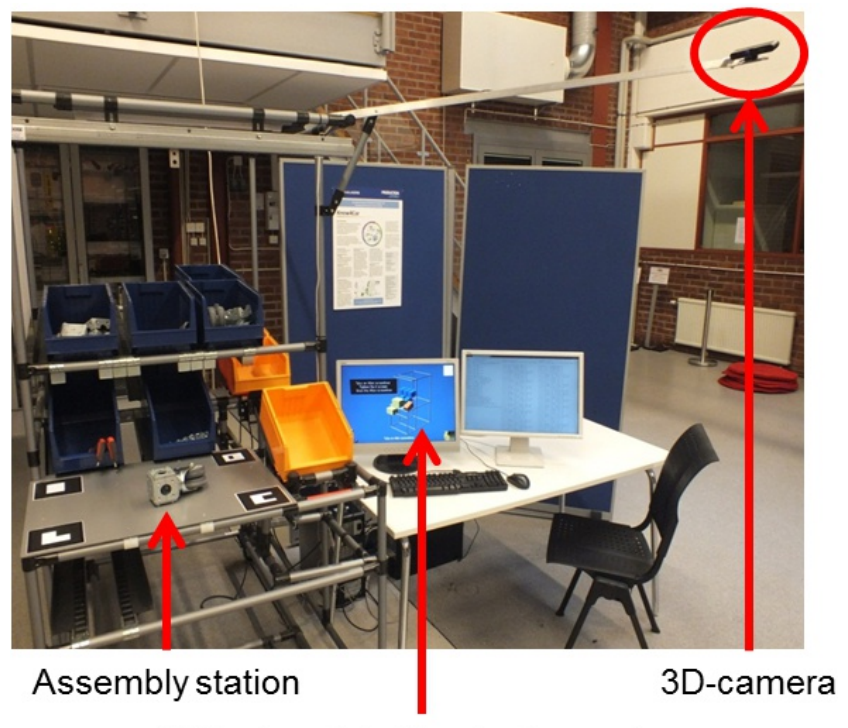

Virtual model of tracked operator

Fig. 4. Illustration of the platform

A simplified virtual model of the assembly station has been modeled using the Unity 3D game development software. Thus, the user interface displayed in Unity 3D fulfills the following features (see Fig. 5):

- Display textual work instructions;

- Animate the virtual model with respect to the work instructions;

- Animate an avatar of the shop-floor operator;

The operator's body is tracked using the 3D camera and her/his avatar is displayed in the virtual environment. The integration of the $3 \mathrm{D}$ camera with the Unity $3 \mathrm{D}$ software is done using Zigfu plugin ${ }^{6}$. Thanks to this integration, the movements of the shop-floor operator and her/his interactions with the real assembly station can be animated in real-time in the virtual model.

The interaction between the real station and the virtual model is done using collision detection in the virtual model. Each "box" of the virtual model represents a 3D

6 http://zigfu.com. Zigfu plugin for Unity. Last visited: 2013-02-27

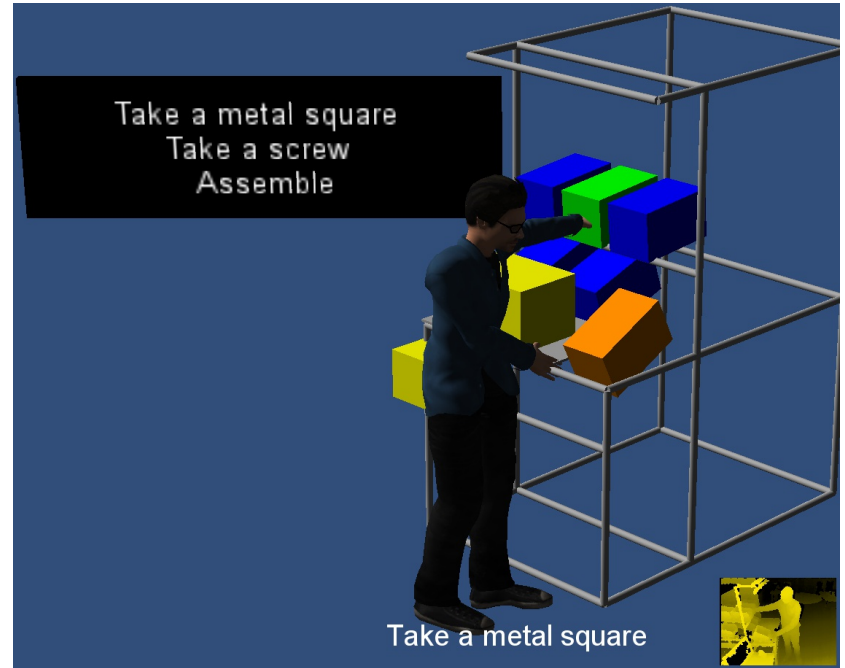

Fig. 5. Capture of the User-Interface

zone in the real world. For instance, a virtual box can be used to represent a real box (e.g. blue and orange boxes) or any 3D area (e.g. the assembly zone in front of the shop-floor operator). Then, if the hand of the operator is located in 3D zone that corresponds to a virtual "box", then a collision is detected by the Unity 3D software. Thus, this solution permits to detect the actions the shop-floor operator is performing in the real world without any onbody sensor nor sensor on the physical objects.

\subsection{Discussion}

The first evaluation exhibits that the use of static cameras permits to obtain a better observation of the assembly station than a head-mounted camera. Moreover, static cameras do not interfere with the movements and the natural field-of-view of the shop-floor operator, and do not require the operator to wear special equipment and batteries. As a consequence, for hand tracking and performance measurements, the use of static cameras is recommended over one head-mounted camera.

The second evaluation exhibits that existing commercial solutions developed by the game industry can efficiently be 
reused in the context of augmented reality applications in assembly industry. As a comparison, there is no affordable real-time application for tracking of human movements using multiple 2D cameras. Another advantage of 3D camera is that only one camera is needed to capture the position of the operator's hand in the $3 \mathrm{D}$ space.

For those reasons, the second set-up, using one 3D camera, has been chosen for the further development of this platform. An application to real-time instructions and information feedback will be presented in the section 5 .

\section{ON THE RELEVANCE OF WORK INSTRUCTIONS - EXPERIMENT ON WORK-FLOW ANALYSIS}

In order to evaluate the importance of feedback information during manual assembly process, an experiment has been conducted using the first set-up with $2 \mathrm{D}$ cameras. This set-up was chosen for that experiment because it permits to record the scene from several points-of-view and then ease later manual analysis of the results. The ability to replay videos was important in that experiment since the aim is to analyze incorrect and unexpected movements.

This experiment has been conducted with 12 participants. Each participant was asked to assemble a product composed of four parts and eight screws (Figure 6). Each participant was asked to assemble this product, first without work instructions, and then, with paper-based static work instructions.
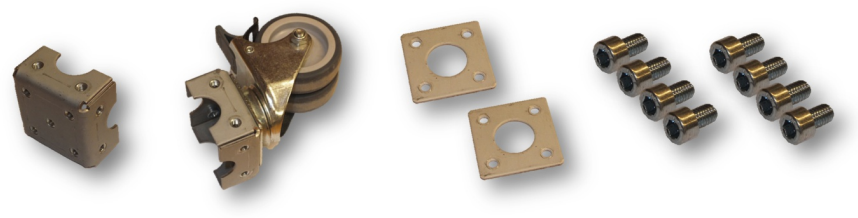

Fig. 6. Parts of the product to be assembled

The product was simple enough so that no long learning phase was required. Before the first run, each participant was given one correctly assembled product and the parts to assemble; (s)he was allowed to observe and try to assemble it during five minutes. The participants were then asked to assemble the product at a normal pace (as if they were asked to perform this operation many times a day). Before the second run, the paper-based instructions were placed on each side of the assembly station, and the participant was allowed to read the instructions for up to five extra minutes.

Once the two runs were done, the products were inspected. The first quality test refers to the correct orientation of the two main parts (Figure 7). The second quality test refers to the correct amount of parts (e.g. some participants put up to four metal squares instead of two).

The table 1 gives the results of this experiment. Based on the results, this experiment permits to conclude that:

- Instructions reading is time consuming, even though some instructions can be read whilst performing an operation.
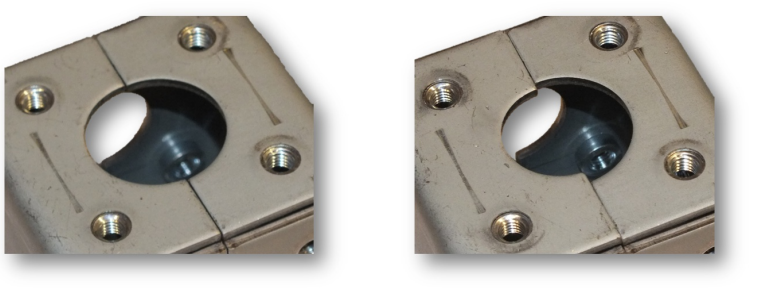

Fig. 7. Correct orientation (left) and incorrect orientation (right)

- Instructions permit to improve the product quality. However, there is still an important gap.

- Instructions can help to improve ergonomic working positions. In this case, the ergonomics criterion was analyze afterwards using the video replay. This criterion refers to the way the operator handles the product during the assembly.

Table 1. Results of the experiment

\begin{tabular}{|c|c|c|}
\hline & Without instructions & With instructions \\
\hline \hline Assembly time & $1 \mathrm{~min} 49 \mathrm{sec}$ & $1 \mathrm{~min} 55 \mathrm{sec}$ \\
\hline \hline Quality test 1 & $42 \%$ & $92 \%$ \\
\hline Quality test 2 & $75 \%$ & $83 \%$ \\
\hline \hline Ergonomics 1 & $71 \%$ & $100 \%$ \\
\hline
\end{tabular}

In addition, the detailed analysis of the videos also exhibits that, in average, only $81 \%$ of the instructions were followed by the participants; this rate varies from $27 \%$ to $100 \%$.

Real-time work instructions would permit to increase relevance of the instruction which is currently displayed and then have more impact on the operator's attention.

Tracking of hands movement would permit to detect missing or erroneous movements, and then, coupled with online feedback information, would reduce the rate of badquality products.

As a conclusion, the application of the platform presented in section 3.3 in an industrial context is then expected to improve the product quality by the reduction of internal rejects. Indeed, most of internal rejects (wrong part assembled, part missing, extra component added. (See Bäckstrand [2009])) could be avoided if the shop-floor operator were provided with online feedback information.

\section{APPLICATION TO REAL-TIME INSTRUCTION AND PERFORMANCE MEASUREMENT}

Based on the results presented in the previous section, this section presents how a body movements' tracking system using a 3D camera and video game user interface can be integrated in an automation system. Real-time instructions can then be retrieved from a knowledge platform; current status and performance measurements can also be updated to the same platform.

\subsection{Modeling of operations using pre- and post-conditions}

The sequences of operations that should be performed to assemble a product can be modeled using pre- and postconditions. A pre-condition defines the set of necessary 
conditions that should be fulfilled to start an operation; while a post-condition defines the set of necessary conditions that should be fulfilled to complete an operation. Using these conditions, a finite automaton composed of three states can be defined in order to model the behavior of an operation. An operation could be either in its initial state $\left(s_{i}\right)$, execution state $\left(s_{e}\right)$, or final state $\left(s_{f}\right)$. The pre-condition permits to evolve from the initial state to the execution state, and the post-condition permits to evolve from the execution state to the final state. For further details about this modeling the reader is referred to Lennartson et al. [2010].

In the context of manual assembly, the conditions to evolve from one state of an operation to the next one can be defined by a set of movements or interactions between the shop-floor operator and the assembly station.

For the experiment presented in this paper, the pre- and post-conditions are associated to interactions between the shop-floor operator's hands and a zone of the assembly station. As presented in section 3.3, these interactions can be detected using virtual collisions. For example, the precondition "LeftHand Enter BoxH1" corresponds to an event that will be emitted when the left hand of the shopfloor operator enters the box labeled BoxH1 in the virtual model. The introduction of a finite automaton model also permits to model more complex pre- or a post-conditions such as sequences of events or alternatives. The figure 8 illustrates three examples:

(1) a simple pre-condition (LeftHand Enter BoxH1) and a simple post-condition (LeftHand Exit BoxH3);

(2) a simple pre-condition (always True) and an alternative post-condition ((LeftHand OR RightHand) Exit ВохH3);

(3) a simple pre-condition (always True) and a sequential post-condition ((LeftHand AND RightHand) Exit ВохH3).

\subsection{Architecture and communication}

In order to be flexible and to permit interoperability with existing subsystems, the architecture has been defined using multiple agents. Each agent has a specific role; the communication between several agents permits to share data and information. The current architecture is composed of the following major agents:

- Scenario agent: this agent retrieves work instructions from the knowledge platform and updates performance measurements to the same knowledge platform.

- User-Interface agent: this agent displays the work instructions and the feedback information to the shopfloor operator. This agent also permits to capture the interaction between the shop-floor operator and the assembly station.

- Performance measurement agent: based on the data received from the User-Interface agent, this agent computes various performances measurements (operation duration, correct movements ratio, ....).

- Message broker agent: this agent is responsible for the routing of the message between the different agents.
(1)
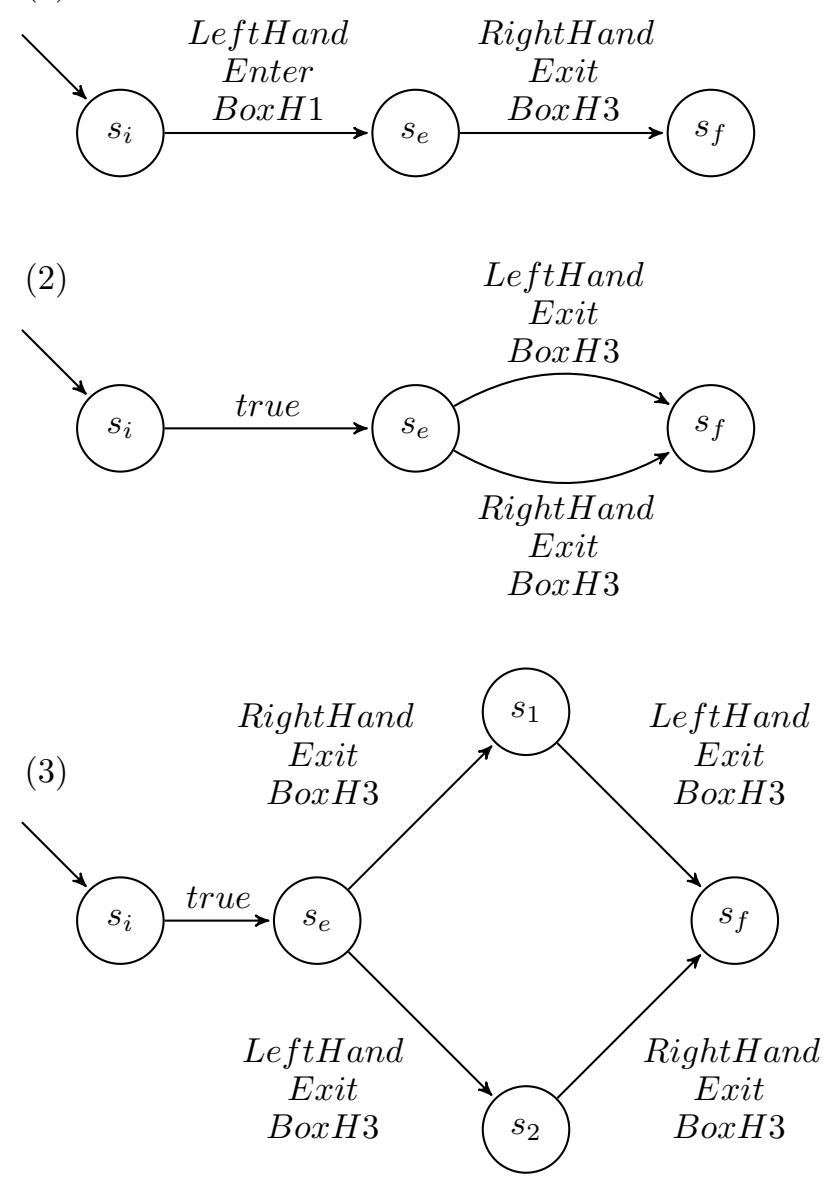

Fig. 8. Example of state models

\subsection{Results}

The real-time detection of the interactions between the shop-floor operator and the assembly station permits to improve the supervision of the actual operations workflow. Indeed, each operation (e.g. pick a part from the top-left box) can be decomposed into a set of movements that can be detected through virtual collision (e.g. the left hand enters the top-left box, the left hand exits the top-left box, the left hand enters the assembly zone...). Thanks to this real-time detection, real-time work instructions can be displayed to the shop-floor operator. This permits to reduce the amount of work instructions that is displayed at a time and to increase the relevance of these works instructions: the instructions that are displayed correspond to the operation that the shop-floor operator is currently doing and/or the few following ones. The figure 9 gives an illustration of this user interface.

The real-time detection of these interactions also permits to provide online feedback information regarding the success or failure of the current operation. For example, if the operator should pick a part from the top-left box, then this box is highlighted in the virtual model (the box is displayed in green (see Fig. 9) and is blinking); if the operator puts her/his hand in another box, that box will be displayed in red. In order to evaluate the performance of a shop-floor operator - especially during training sessions and ramp-up phases - all triggered move- 
ments are time-stamped. Based on these raw data, several time-related measurements can be defined (total assembly duration, duration for specific operation...). If the shopfloor operator is supposed to follow a predefined work-flow, measurements related to inaccurate or wrong movements can also be defined (ratio correct/wrong movements...).

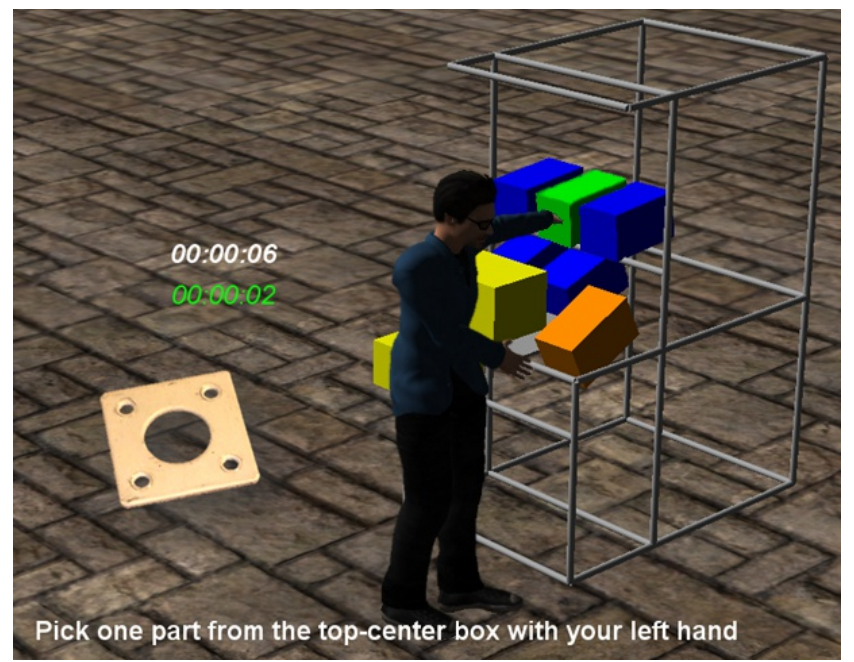

Fig. 9. Capture of the final User-Interface

A demonstration of this experiment is available on the Know4Car's Youtube account ${ }^{7}$. In this demonstration, the only measurement displayed to the shop-floor operator is the duration of the current operation and its comparison to the expected duration. Dynamic work instructions are displayed both in a graphical and in a textual ways.

\section{ON ETHICAL ASPECTS AND USER-ACCEPTANCE}

While a better observation of shop-floor operators' actions permits a better integration with the automation part of a system, it also implies that performance measurement can be performed using these observed actions. As it has already been studied in numerous papers (Fugger et al. [2007], Mahoney et al. [2007], Allen et al. [2007], Sewell and Barker [2006], Petronio [2002], Stanton and Stam [2002], Ziebell and Singh [2001], Mason et al. [2002], Miller and Weckert [2000]), the measurement of human performance on the workplace is a sensitive issue. As all surveillance means, the measurement of human performances can be used as a form of caring or as a form of coercion (Sewell and Barker [2006]).

In the first case: caring surveillance, the performance measurements are willing to help and protect the employee. For instance, surveillance permits to detect errors earlier and then reduce their induced cost; small mistakes would then have less impact on the global appreciation of the employee efficiency.

In the second case: coercive surveillance, the performance measurements mainly benefit the employer. Performance measurements are designed to minimize the opportunities for employees to avoid working as hard as they really can.

\footnotetext{
7 http://www.youtube.com/watch?v=7KiaBsGDmfU

http://www.youtube.com/watch?v=XAuXAvphYOY
}

The correct definition of the level of care/coercion is important since the user acceptance has a huge impact on the success of the performance measurement method. Thus, the user acceptance increases if employees perceive some benefit. Allen et al. [2007] mentioned that "privacy issues may appear less salient and resistance less likely when employees believe electronic surveillance demonstrates a form of caring rather than an excessive form of coercive control that primarily benefits employers".

Below are listed some recommendations regarding human performance measurements that can be applied in the context of manual assembly:

(1) Performance measurements should be made on an individual level, not on a team level.

For instance, if the performance of a team is average, some individuals may work really hard and are not rewarded as much as expected, while other individuals may take advantage of the good performance of the previous ones.

(2) Performance measurements should only depend on the performance of the human operator.

The throughput of one station can easily be measured; however it can be highly dependent on the input flow - which could depends on the performance of a machine - and not only on the human operator performance. A higher level of observation permits to identify these external factors.

(3) Feedback information should be displayed as soon as possible.

The sooner the better: early notification of small deviations facilitates the corrections of these deviations and reduces their impact on the cost of the final product.

According to Allen et al. [2007], Sewell and Barker [2006], Petronio [2002], surveillance is more accepted in the USA than in Germany and Great Britain for instance. One can also wonder if resistance to new IT technologies and surveillance is mainly due to resistance to new technologies or to lack of trust in their hierarchy.

As a conclusion, the choice and the definition of performance measurements should involve the partners from the different teams and areas. Sharing the knowledge and involving blue collars employees in the decision process would also increase acceptance of measurements and decisions.

\section{CONCLUSION AND PROSPECTS}

The experimental results presented in this paper have permitted to select a set-up that can be used in an industrial environment in order to improve the integration of human and automation. These experimental results also demonstrate that the technology development impulsed by the video-game industry can not only benefit to Virtual Reality applications but also to Augmented and Mixed Reality applications.

On-going work consider the definition of more advanced measurements that can be used during training sessions and ramp-up phases, and the adaptation of work instructions according to individual performance. 


\section{ACKNOWLEDGEMENTS}

This work has been supported by the Know4Car project, grant agreement number 284602, funded by the EC Seventh Framework Programme theme FoF-ICT-2011.7.4.

\section{REFERENCES}

Myria Watkins Allen, Stephanie J Coopman, Joy L Hart, and Kasey L Walker. Workplace surveillance and managing privacy boundaries. Management Communication Quarterly, 21(2):172-200, 2007.

Gunnar Bäckstrand. Information flow and product quality in human based assembly. $\mathrm{PhD}$ thesis, Loughborough University, 2009.

Dima Damen, Andrew Gee, Walterio Mayol-Cuevas, and Andrew Calway. Egocentric real-time workspace monitoring using an rgb-d camera. In Proc. IEEE/RSJ Int. Conf. on Intelligent Robots and Systems (IROS), 2012.

Francesca De Crescenzio, Massimiliano Fantini, Franco Persiani, Luigi Di Stefano, Pietro Azzari, and Samuele Salti. Augmented reality for aircraft maintenance training and operations support. Computer Graphics and Applications, IEEE, 31(1):96-101, 2011.

Erwin Fugger, Barbara Prazak, Sten Hanke, and Siegfried Wassertheurer. Requirements and ethical issues for sensor-augmented environments in elderly care. Universal Acess in Human Computer Interaction. Coping with Diversity, 4554:887-893, 2007. Lecture Notes in Computer Science.

Richard M Greenough and Benny Tjahjono. An interactive electronic technical manual for an advanced aerospace assembly machine. The International Journal of Advanced Manufacturing Technology, 33(9):10451055, 2007.

Steven Henderson and Steven Feiner. Exploring the benefits of augmented reality documentation for maintenance and repair. Visualization and Computer Graphics, IEEE Transactions on, 17(10):1355-1368, 2011.

Tim N. Höffler and Detlev Leutner. Instructional animation versus static pictures: A meta-analysis. Learning and instruction, 17(6):722-738, 2007.

S. Iliano, V. Chimienti, and G. Dini. Training by augmented reality in industrial environments: a case study. In CIRP 4th Conference on Assembly Technologies and Systems, 2012.

B. Lennartson, K. Bengtsson, C. Yuan, K. Andersson, M. Fabian, P. Falkman, and K. Ảkesson. Sequence planning for integrated product, process and automation design. IEEE Transactions on Automation Science and Engineering, 7(4):791-802, 2010.

C. Lenz, M. Grimm, T. Röder, and A. Knoll. Fusing multiple kinects to survey shared human-robot-workspaces. Technical Report Technical Report TUM-I1214, Technische Universität München, Munich, Germany, 2012.

Diane F Mahoney, Ruth B Purtilo, Frank M Webbe, Majd Alwan, Ashok J Bharucha, Tim D Adlam, Holly B Jimison, Beverly Turner, and S Becker. In-home monitoring of persons with dementia: Ethical guidelines for technology research and development. Alzheimer's and Dementia, 3(3):217-226, 2007.

David Mason, Graham Button, Gloria Lankshear, Sally Coates, and Wes Sharrock. On the poverty of apriorism: technology, surveillance in the workplace and employee responses. Information, Communication \& Society, 5 (4):555-572, 2002.

Lynn McAtamney and E. Nigel Corlett. Rula: a survey method for the investigation of work-related upper limb disorders. Applied ergonomics, 24(2):91-99, 1993. Online assessment: http://www.rula.co.uk.

Seumas Miller and John Weckert. Privacy, the workplace and the internet. Journal of Business Ethics, 28(3):255265, 2000.

Raja Parasuraman, Thomas B Sheridan, and Christopher D Wickens. A model for types and levels of human interaction with automation. Systems, Man and Cybernetics, Part A: Systems and Humans, IEEE Transactions on, 30(3):286-297, 2000.

Sandra Sporbert Petronio. Boundaries of privacy: Dialectics of disclosure. SUNY Press, 2002.

Graham Sewell and James R Barker. Coercion versus care: Using irony to make sense of organizational surveillance. Academy of Management Review, 31(4):934-961, 2006.

Jeffrey M Stanton and Kathryn R Stam. Information technology, privacy, and power within organizations: A view from boundary theory and social exchange perspectives. Surveillance \& Society, 1(2):152-190, 2002.

Didier Stricker and Gabriele Bleser. From interactive to adaptive augmented reality. In Ubiquitous Virtual Reality (ISUVR), 2012 International Symposium on, pages 18-21. IEEE, 2012.

Jeffrey Too Chuan Tan and Tamio Arai. Triple stereo vision system for safety monitoring of human-robot collaboration in cellular manufacturing. In Assembly and Manufacturing (ISAM), 2011 IEEE International Symposium on, pages 1-6. IEEE, 2011.

Nicolas Vignais, Markus Miezal, Gabriele Bleser, Katharina Mura, Dominic Gorecky, and Frédéric Marin. Innovative system for real-time ergonomic feedback in industrial manufacturing. Applied Ergonomics, 2012.

Yixiao Yun, Irene Yu-Hua Gu, Julien Provost, and Knut Åkesson. Multi-view hand tracking using epipolar geometry-based consistent labeling for an industrial application. In Submitted to the International Conference on Image Analysis and Processing (ICIAP 2013), 2013.

D. Ziebell and B. P. Singh. Final report on leading indicators of human performance. Technical report, EPRI and the U.S. Department of Energy, 2001. 